\title{
Präzision. Individualisiert.
}

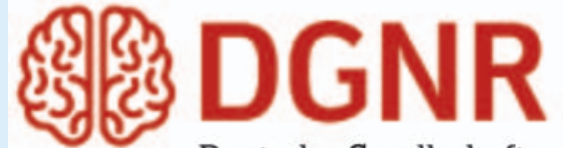

Deutsche Gesellschaft

für Neuroradiologie e.V.

\section{Save the Date und Einladung zur DGNR- Jahrestagung}

\section{2.-14.10.2022 in Kassel}

Sehr geehrte Damen und Herren, liebe Kolleginnen und Kollegen,

im Namen des DGNR-Vorstands und meines Kongressteams in Lübeck laden wir Sie herzlich zur 57. Jahrestagung der Deutschen Gesellschaft für Neuroradiologie e. V. im Oktober 2022 ein!

Nach aktuellem Stand der Lage können wir davon ausgehen, nach 2 digitalen Formaten die kommende Jahrestagung wieder als
Live-Kongress in voller Präsenz ausrichten zu dürfen.

Hierfür haben wir einen Standort gewählt, der dem Wunsch nach guter Anbindung und Erreichbarkeit Rechnung trägt, über ein höchst attraktives Kongresszentrum verfügt und nicht zuletzt einen ansprechenden, kulturellen Rahmen bereitstellt.

So freuen wir uns, dass wir für die 57 . Jahrestagung der DGNR das Kongress-Palais in der documenta-Stadt Kassel als Veranstaltungsort gewinnen konnten. Hier können Sie sich schon einmal einen ersten Eindruck verschaffen: www.kongress-palais.de.

Unser Kongressmotto Präzision. Individualisiert. greift die sich kontinuierlich weiterentwickelnden Themenfelder der patientenspezifischen Therapien in der interventionellen Neuroradiologie auf, fokussiert aber auch all die Softwarelösungen, die Wege und Zeiten zur spezifischen Diagnostik und individualisierten Patientenbehandlung optimieren und die Verbreitung von Innovationen beschleunigen. Präzision. Individualisiert. spiegelt darü- ber hinaus die zunehmend individualisierten Arbeitsweisen in unserem Fachgebiet wider, von der Teleradiologie bis zur Robotik.

Wir freuen uns über Ihre Mitwirkung an der Tagung - als Referentinnen/Referenten, als Einreicherinnen/Einreicher wissenschaftlicher Beiträge und Cases und nicht zuletzt als Besucherinnen/Besucher.

Informationen zum Kongressstandort, zum wissenschaftlichen Programm und Callfor-Abstract, zur Industrie und zum Begleitprogramm finden Sie demnächst auf www.neurorad.de.

Mit kollegialen Grüßen

Prof. Dr. med. Peter Schramm

Direktor des Instituts für Neuroradiologie Universitätsklinikum Schleswig-Holstein Campus Lübeck

Kongresspräsident 2022

Prof. Dr. med. Claus Zimmer

Direktor der Abteilung für Neuroradiologie am Klinikum rechts der Isar der TUM

Präsident der DGNR 